\title{
Simulation and Random Vibration Test of Structural Dynamic Characteristics of Vacuum Tube
}

\author{
Haijun Mo ${ }^{1, a}$, Huarong Qiu ${ }^{1, b}$ \\ ${ }^{1}$ School of Mechanical and Automotive Engineering, South China University of Technology, \\ Guangzhou, 510640, China \\ aemail: mohj@scut.edu.cn, bemail: huayunq@163.com
}

Keywords: Vacuum tube; Modal analysis; Random vibration analysis; Random vibration test

\begin{abstract}
Vacuum tube is working in a very harsh conditions, a high requirement for structural reliability has to be put forward. The research method of structural dynamic characteristics analysis was discussed in this paper, and the method combined finite element simulation and random vibration test is used to analyze the dynamic characteristics of the vacuum tube. The natural vibration characteristics of the vacuum tube and vibration response in the working conditions were studied. The results show that the simulation analysis was basically consistent with the random vibration test and the vacuum tube appears resonance in working conditions.
\end{abstract}

\section{Introduction}

In this paper, the vacuum tube is mainly used in the field of transport, the working environment is usually very bad. The vacuum tube may be subjected to various dynamic loads, which may cause its structural deformation and even cause resonance, and lead to deterioration or failure of electrical performance. In order to guarantee the reliability of the structure dynamics, it is necessary to study the dynamic characteristics of the structure[1-3]. Dynamic characteristics usually refer to the vibration characteristics of the structure and the vibration response under the external load[4]. The vibration characteristics of structure mainly include modal frequency and modal shapes, which is important parameters for guiding the structural design to avoid resonance. The modal analysis is used to calculate the vibration characteristics of the structural system, and it can be used as the starting point for other dynamic analysis to obtain the vibration response of the structural system under different loads. Random vibration analysis is a qualitative analysis technique for the response and reliability of structural systems under random loads based on probability and statistics[5]. Transportation tools are more likely to suffer vibration and impact from the ground, air and engine. This kind of vibration cannot be described by mathematical formula and time function, and can only be expressed by statistical methods and data, known as random vibration. Random vibration can be more appropriate to represent the real machinery working environment of the vacuum tube.

In this paper, the modal analysis and random vibration analysis of the vacuum tube are carried out. The inherent vibration characteristics and weakest part, displacement response, load amplification factor under environmental loads are obtained, which guide the anti-vibration design of the vacuum tube.

\section{Theoretical analysis of dynamic characteristics}

The dynamics differential equation of N-DOF linear systems can be expressed as follows:

$$
[M] \ddot{U}(t)+[C] \dot{U}(t)+[K] U(t)=F(t)
$$

For the non-damping free vibration system, $F(t)=0,[C]=0$, Formula (1) can be simplified as the modal equation:

$$
[M] \ddot{U}+[K] U=0
$$

For linear systems, the free vibration is simple harmonic motion. Formula (2) can be expressed as follows: 


$$
\left([K]-\omega_{i}^{2}[M]\right) \phi_{i}=0
$$

$\mathrm{N}$ different positive $\operatorname{roots}\left(\omega_{i}(i=1,2, \cdots, n)\right)$ and linearly independent nonzero vector $\left(\phi_{i}\right)$ are obtained from Formula (3). Where $\phi_{i}$ is the vibration mode of the i-th order natural frequency. $\omega_{i}$ is eigenvalue which represents the $i$-th order natural angular frequency. The matrix $[\phi]=\left[\begin{array}{llll}\phi_{1} & \phi_{2} & \cdots & \phi_{n}\end{array}\right]$ is composed of the modal space coordinate system.

Due to the random vibration can't be described by mathematical formula and time function, the concept of auto-power spectral density function is proposed based on probability theory and mathematical statistics. Its physical meaning is the distribution of vibration energy in frequency domain, which is expressed by Wiener-Khintchine formula:

$$
S_{F}(\omega)=\frac{1}{2 \pi} \int_{-\infty}^{\infty} R_{F}(\tau) e^{-i \omega \tau} d \tau
$$

Where: $R_{F}(\tau)$ is autocorrelation function of random process, which can be expressed as follows:

$$
R_{F}(\tau)=E[F(t) F(t+\tau)]=\int_{-\infty}^{\infty} \int_{-\infty}^{\infty} f_{1} f_{2} g\left(f_{1}, f_{2} ; \tau\right) d f_{1} d f_{2}
$$

Where: $g\left(f_{1}, f_{2}\right)$ is two dimensional probability density function of random process.

Formula (1) is a coupling equation included each point's physical coordinates, which cannot be solved directly in the analysis of large-scale engineering structures. By means of the coordinate transformation technology, the physical space coordinates can be transformed into modal space coordinates:

$$
U=\sum_{i=1}^{n} \phi_{i} X_{i}=[\phi] X
$$

Proportional damping is adapted. According to the orthogonality of $[\phi]$ to $[M]$ and $[K]$, the formula (1) can be decoupled as follows:

$$
\ddot{X}+2 \xi \Omega \dot{X}+\Omega^{2} X=Y
$$

Where: $\Omega^{2}=\operatorname{diag}\left[\omega_{j}^{2}\right], j=1,2, \cdots, n$.

By introducing the statistical characteristics of random vibration load, the correlation matrix and spectrum density matrix of the response in the physical space can be obtained:

$$
\begin{gathered}
R_{U}=[\phi] \int_{0}^{\infty} \int_{0}^{\infty} h\left(\lambda_{1}\right)[\phi]^{T} R_{F}\left(\tau+\lambda_{1}-\lambda_{2}\right)[\phi] h\left(\lambda_{2}\right) d \lambda_{1} d \lambda_{2}[\phi]^{T} \\
S_{U}(\omega)=[\phi] H(\omega)[\phi]^{T} S_{F}(\omega)[\phi] H^{*}(\omega)[\phi]^{T}
\end{gathered}
$$

\section{Modal analysis}

The 3D Solid Model of vacuum tube was established in Pro/e and imported into ANSYS after appropriate simplification. In ANSYS, the material properties and the contact relation of each part and the meshing are defined. The finite element model is shown in Figure 1. The number of nodes in the finite element model is 1119649 , and the number of elements is 453366 .

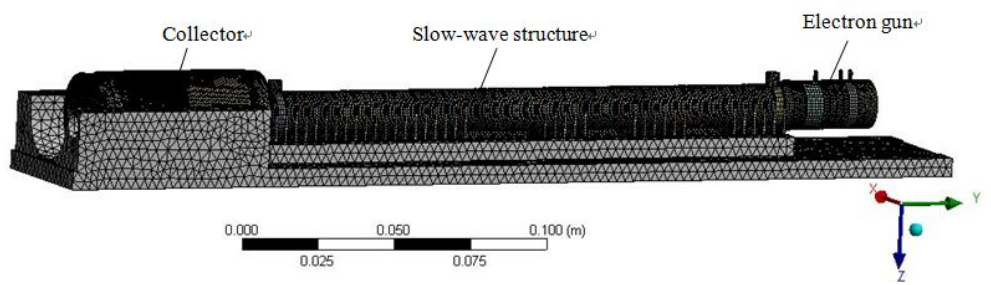

Fig. 1 FE Model of vacuum tube

According to the actual installation of the vacuum tube, the fixed constraint is applied to the screw hole of the bottom plate. The first four modal of the vacuum tube are obtained by the method 
of Lanczos Block. The first four modal frequencies are shown in Table 2 and figure 1 is the modal shapes.

Tab.1 The First Four Modal Frequencies of vacuum tube

\begin{tabular}{|c|c|c|c|c|}
\hline Order & 1 & 2 & 3 & 4 \\
\hline Modal frequency/Hz & 1540.8 & 1944.4 & 2436.4 & 2951.5 \\
\hline 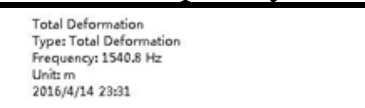 & & 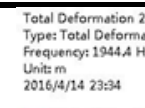 & & \\
\hline 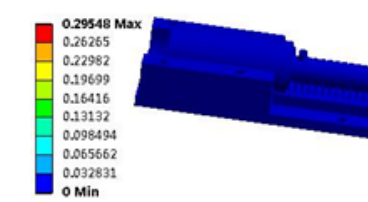 & & 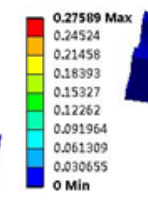 & & \\
\hline 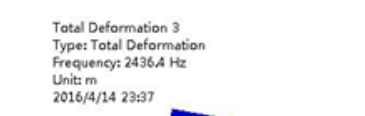 & & $\begin{array}{l}\text { Total Deformetion } \\
\text { Type: Total Deform, } \\
\text { Frequency: } 2951.5 \\
\text { Unit: } m \\
\text { 2016/4/14 23:38 }\end{array}$ & & \\
\hline 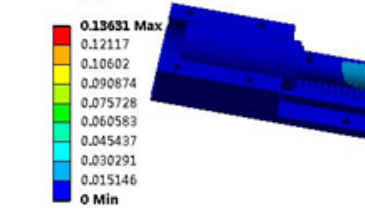 & & 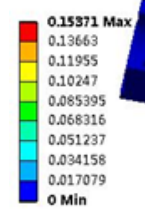 & & \\
\hline
\end{tabular}

Fig. 2 The First Four Modal Shapes of vacuum tube

The results of modal analysis show that the low order modes of the vacuum tube are mainly focused on the electron gun and the slow-wave structure. The vibration mode is mainly manifested by the vibration of the electron gun along the $\mathrm{X}$ and $\mathrm{Z}$ direction, and the slow wave structure along the $\mathrm{X}$ direction.

\section{Random vibration analysis}

According to theoretical analysis of the first section, the modal superposition method is used to solve random vibration, and the boundary conditions are consistent with the modal analysis. The vacuum electron tube has two order modes in the $2000 \mathrm{~Hz}$, which is in the frequency range of environmental load. In order to study the influence of resonance on the $\mathrm{X}$ direction and $\mathrm{Z}$ direction, the random vibration analysis is carried out. According to requirements of the vacuum tube's ambient vibration test, the power spectral density (PSD) is applied to the fixed constraint on the screw hole of the vacuum tube in the random vibration analysis. The PSD is shown in Figure 3, and its root mean square (RMS) is $14.2 \mathrm{~g}$.

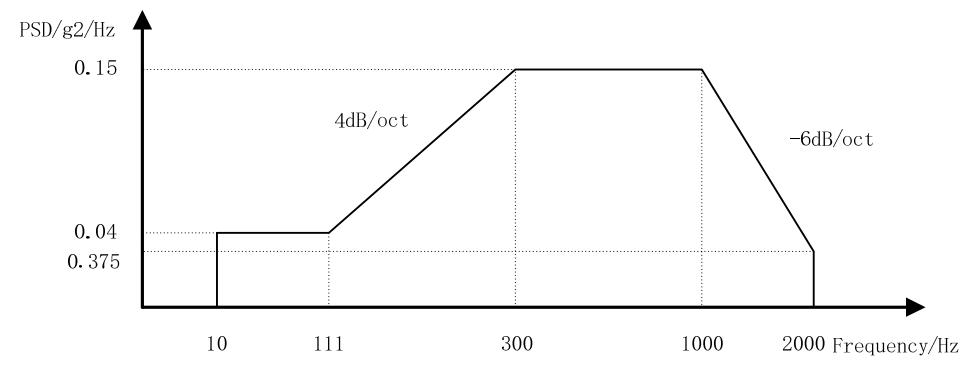

Fig.3 Power density spectrum

By the theory of random vibration, it is known that damping is essential for dynamic response analysis of structures. Proportional damping model is adopted in this paper. The viscous damping ( $\alpha$ damping) is always be ignored and the structural damping is only considered ( $\beta$ damping) in dynamics analysis by ANSYS Workbench. The structural damping can be obtained through modal damping ratio $\xi_{i}$ and angular frequency $\omega_{i}[4]$.

$$
\beta=\frac{2 \xi_{i}}{\omega_{i}}
$$

In this paper, the damping ratio is selected as $1 \%$ based on the relevant analysis[6]. $\omega_{i}$ is 
response frequency $(\mathrm{rad} / \mathrm{sec})$ and $\omega_{i}=2 \pi f_{i}$, which is related to the structural first-order natural frequency. According to modal analysis results, the structural damping value of this gun is $2.0783 \mathrm{e}-6$.

After setting load and damping, the response results of the vacuum tube under random vibration excitation are calculated. Because of aperiodic and randomness of random vibration, the analysis results can only be described by probability statistics method. Figure 4 and figure 5 show the distribution of stress and deformation during random vibration analysis on the X direction in $3 \sigma$.

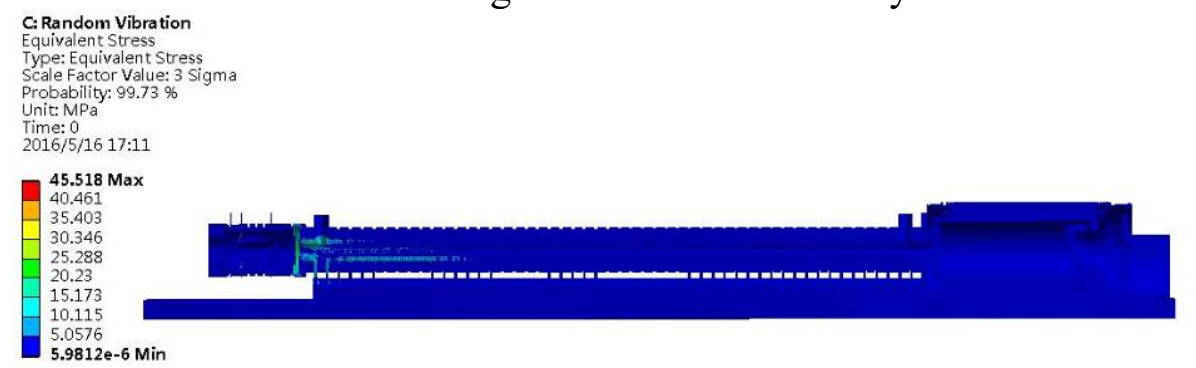

Fig.4 The distribution of stress

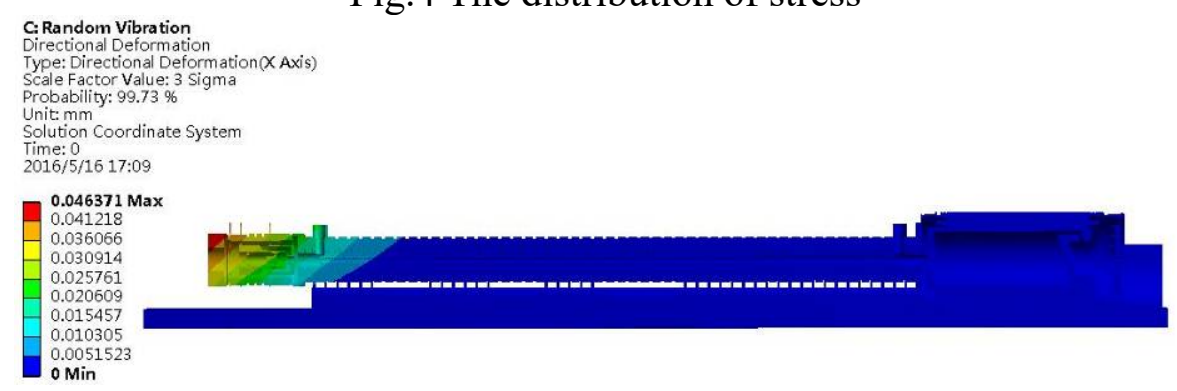

Fig.5 The distribution of deformation

Through the analysis, when the vacuum tube is subjected to random vibration excitation on the $\mathrm{X}$ direction, the maximum stress is located inside the electron gun, and the maximum value is $45.52 \mathrm{MPa}$. The maximum displacement is $0.046 \mathrm{~mm}$, which is located at the end of the electron gun. The deformation directly affects the direction of electron injection into the slow wave structure. When the vacuum tube is subjected to random vibration excitation on the $\mathrm{Z}$ direction, the maximum stress and displacement of the electron gun is $42.36 \mathrm{MPa}$ and $0.021 \mathrm{~mm}$. Overall results show that anti-vibration performance of the vacuum tube on $\mathrm{X}$ direction is weaker than that on $\mathrm{Z}$ direction.

\section{Random vibration test}

Because the vacuum tube structure is small and it is difficult to be excited, the method of collecting vibration response signals can't be used by acceleration sensor. In this paper, a non-contact vibration test system is built by laser doppler vibrometer and Vibration Test-table. Random vibration test analysis mainly contains the excitation, acquisition of excitation signal and response signal and signal analysis, design of random vibration test analysis and random vibration test platform as shown in figure 6. In Figure 6, the accelerometer B is used to measure the acceleration signal of vibration table, and feed back to vibration controller, so that the power spectrum density (PSD) of random vibration excitation is controlled within the range of requirement. The accelerometer $\mathrm{A}$ is used to measure the random vibration excitation of actual loading on the vacuum tube. The vibration response in time-domain and frequency-domain of the vacuum tube can be observed by the laser doppler vibrometer.

In order to carry out the random vibration test of $\mathrm{X}$ direction, 33 measuring points are set on surface of the slow wave structure and the electron gun. Measuring point number from left to right is 1-33, where points 1-27 are located in the slow wave structure, and the points 28-33 are located in the electron gun. The distribution of measuring points is shown in Figure 7. 


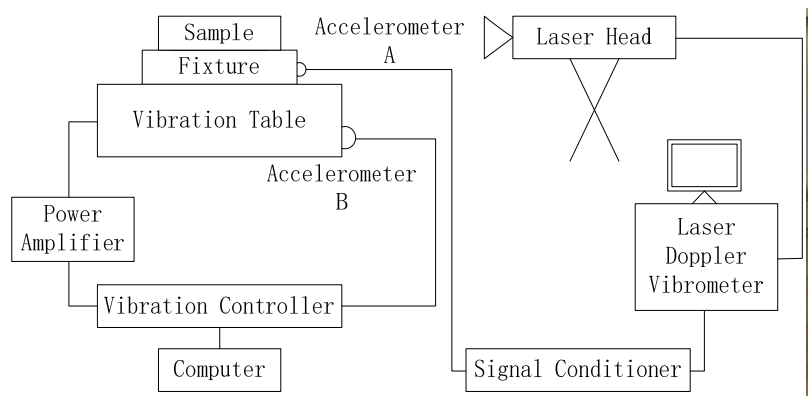

(a)Design of Random Vibration Test Analysis

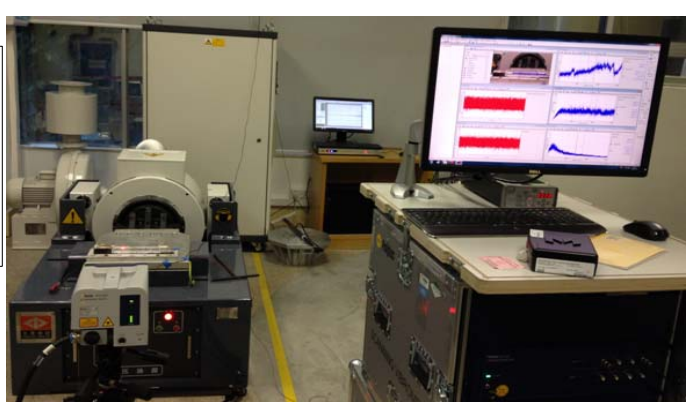

(b)Random Vibration Test Platform

Fig.6 Random Vibration Test System

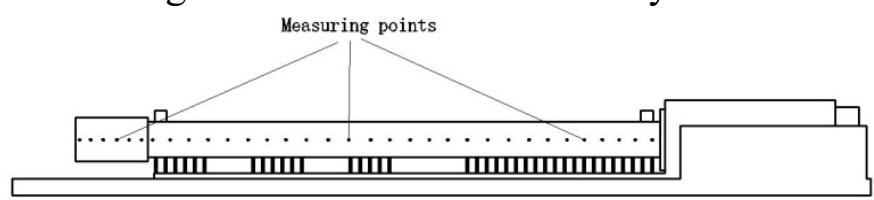

Fig.7 The distribution of measuring Points

The root mean square (RSM) of each measurement point is tested, and it can be known that the power spectral density of actual vibration excitation is $13.0 \mathrm{~g}$ through accelerometer $\mathrm{A}$. The magnification of RMS of each measuring point under random vibration excitation can be calculated. The root mean square (RMS) of each measuring point and its amplification are compared with the simulation results as shown in figure 8 .

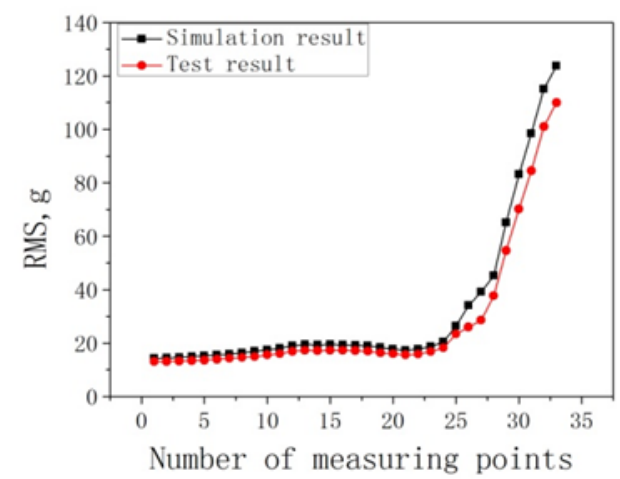

(a) The comparison of RMS

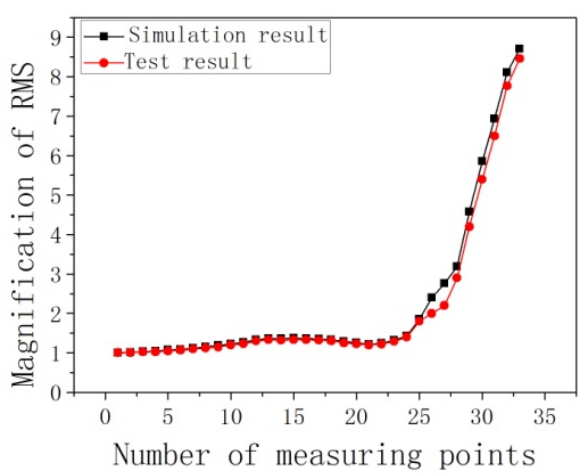

(b) The comparison of RMS's magnification

Fig. 8 The comparison of RMS and RMS's magnification

From figure 8 (a), we can know that the RMS of the simulation is slightly larger than the test. The main reason is that the RMS of the actual vibration excitation is $13.0 \mathrm{~g}$, which is lower than the $14.2 \mathrm{~g}$ of the ideal vibration excitation. From Figure 8 (b), we can know that the RMS's amplification of the simulation is basically consistent with the test. The above analysis shows that the random vibration simulation results of the vacuum tube are reliable. From figure 8, we can find that the change of RMS and its amplification of measuring points from number 0 to 25 is very small. This section belongs to the slow wave structure, the results show that the slow wave structure will not appear resonance under the random vibration load of the frequency from 0 to $2000 \mathrm{~Hz}$. The RMS and its amplification of measuring points from number 26 to 33 are gradually increasing. This section mainly belongs to the electron gun, the results show that the section will appear resonance under the random vibration load. The maximum value of the RMS's amplification of the electron gun is 8.46 , which will seriously affect the service life of the vacuum tube.

\section{Conclusion}

In this paper, the method combined finite element simulation and random vibration test is used to analyze the dynamic characteristics of the vacuum tube, and the natural vibration characteristics and vibration response in working conditions are obtained. By means of finite element software, the modal analysis and random vibration analysis of the vacuum tube are carried out. The first four 
natural frequencies and the vibration modes, the distribution of stress and deformation under the random vibration load are obtained. The results show that the vacuum electron tube appears resonance in the working conditions, and anti-vibration performance of the vacuum tube on $X$ direction is weaker than that on $\mathrm{Z}$ direction. The random vibration test platform is set up, and the RMS and its amplification of each measuring point are obtained under random vibration excitation. After comparative analysis, the simulation results are confirmed to be reliable.

\section{References}

[1] Raina Sushil, Santra Mrityunjay. Analytical Determination of Thermal and Vibrational Performance of Coupled Cavity TWT's [C] . Vacuum Electronics Conference, Costa Mesa, 2000.

[2] Xu Chang-you, Zhao Shi-ke, Hao Bao-liang. Simulation and random vibration test of structural dynamic characteristics of microwave window of traveling wave tube $[\mathrm{J}]$. Chinese Journal of Vacuum Science and Technology, 2013, 33(4): 321-325.

[3] Wang Juan,Wu Gang,Su Xiao-gang. Random vibration analysis of grid-controlled electron gun in TWT[J]. Vacuum Electronics, 2012, (5): 62-65.

[4] Xu Jing-jing. ANSYS 13.0 Workbench numerical simulation technology [M]. Beijing: China WaterPower Press, 2012.

[5] Wen Bang-chun. Theory of Mechanical Vibration and Its Application [M]. Beijing: Higher Education Press, 2009.

[6] Xu Sha, Thermo-Mechanical Coupling Characteristic Analysis and Optimal Design of the Electron Gun of Travelling Wave Tube [D]. South China University of Technology, 2014. 